\title{
A Relationship Between the Major Index For Tableaux and the Charge Statistic For Permutations
}

\author{
Kendra Killpatrick \\ Pepperdine University \\ Malibu, California \\ Kendra.Killpatrick@pepperdine.edu
}

Submitted: Jul 13, 2005; Accepted: Aug 30, 2005; Published: Sep 5, 2005

Mathematics Subject Classifications: 05A15, 05E10

\begin{abstract}
The widely studied $q$-polynomial $f^{\lambda}(q)$, which specializes when $q=1$ to $f^{\lambda}$, the number of standard Young tableaux of shape $\lambda$, has multiple combinatorial interpretations. It represents the dimension of the unipotent representation $S_{q}^{\lambda}$ of the finite general linear group $G L_{n}(q)$, it occurs as a special case of the Kostka-Foulkes polynomials, and it gives the generating function for the major index statistic on standard Young tableaux. Similarly, the $q$-polynomial $g^{\lambda}(q)$ has combinatorial interpretations as the $q$-multinomial coefficient, as the dimension of the permutation representation $M_{q}^{\lambda}$ of the general linear group $G L_{n}(q)$, and as the generating function for both the inversion statistic and the charge statistic on permutations in $W_{\lambda}$. It is a well known result that for $\lambda$ a partition of $n, \operatorname{dim}\left(M_{q}^{\lambda}\right)=\Sigma_{\mu} K_{\mu \lambda} \operatorname{dim}\left(S_{q}^{\mu}\right)$, where the sum is over all partitions $\mu$ of $n$ and where the Kostka number $K_{\mu \lambda}$ gives the number of semistandard Young tableaux of shape $\mu$ and content $\lambda$. Thus $g^{\lambda}(q)-f^{\lambda}(q)$ is a $q$-polynomial with nonnegative coefficients. This paper gives a combinatorial proof of this result by defining an injection $f$ from the set of standard Young tableaux of shape $\lambda, S Y T(\lambda)$, to $W_{\lambda}$ such that $\operatorname{maj}(T)=\operatorname{ch}(f(T))$ for $T \in S Y T(\lambda)$.
\end{abstract}

Key words: Young tableaux, permutation statistics, inversion statistic, charge statistic, Kostka polynomials.

\section{Introduction}

For $\lambda$ any partition of $n, f^{\lambda}$ gives the number of standard Young tableaux of shape $\lambda$. The $q$-version of $f^{\lambda}$ is a polynomial that has many important combinatorial interpretations. In particular, $f^{\lambda}(q)$ is known to give the dimension of the unipotent representation $S_{q}^{\lambda}$ 
of the finite general linear group $G L_{n}(q)$. The polynomial $f^{\lambda}(q)$ can be computed as the generating function for the major index $\operatorname{maj}(T)$ on the set of standard Young tableaux of shape $\lambda, S Y T(\lambda)$.

$$
f^{\lambda}(q)=\sum_{T \in S Y T(\lambda)} q^{\operatorname{maj}(T)}
$$

In addition, the $q$-multinomial coefficient

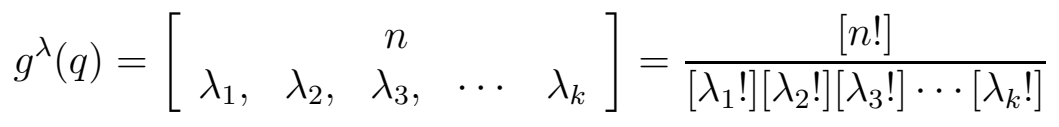

is known to give the dimension of the permutation representation $M_{q}^{\lambda}$ of $G L_{n}(q)$. The polynomial $g^{\lambda}(q)$ also has a combinatorial interpretation as

$$
g^{\lambda}(q)=\sum_{\pi \in W_{\lambda}} q^{i n v(\pi)}
$$

where $W_{\lambda}$ is the subset of permutations in $S_{n}$ of type $\lambda$ and $i n v(\pi)$ is the inversion statistic on $\pi$. The following is a well-known result on the representation of $G L_{n}(q)$ :

Proposition 1. For $\lambda$ a partition of $n$,

$$
\operatorname{dim}\left(M_{q}^{\lambda}\right)=\sum_{\mu \vdash n} K_{\mu \lambda} \operatorname{dim}\left(S_{q}^{\mu}\right)
$$

where $K_{\mu \lambda}$ is the Kostka number which counts the number of semi-standard tableaux of shape $\mu$ and content $\lambda$.

Thus we have

$$
g^{\lambda}(q)=\sum_{\mu \vdash n} K_{\mu \lambda} f^{\mu}(q)
$$

and in particular, since $K_{\lambda \lambda}=1$ for all $\lambda$,

$$
g^{\lambda}(q)=f^{\lambda}(q)+\sum_{\substack{\mu \vdash n \\ \mu \neq \lambda}} K_{\mu \lambda} f^{\mu}(q)
$$

Thus

$$
g^{\lambda}(q)-f^{\lambda}(q)=\sum_{\substack{\mu \vdash n \\ \mu \neq \lambda}} K_{\mu \lambda} f^{\mu}(q)
$$

is a $q$-polynomial with non-negative coefficients. This implies that

$$
g^{\lambda}(q)-f^{\lambda}(q)=\sum_{\pi \in W_{\lambda}} q^{i n v(\pi)}-\sum_{T \in S Y T(\lambda)} q^{\operatorname{maj}(T)}
$$

is a $q$-polynomial with non-negative coefficients. It is natural, then, to seek an injection from standard Young tableaux of shape $\lambda$ to permutations in $W_{\lambda}$ which takes the statistic 
$\operatorname{maj}(T)$ to the statistic $i n v(\pi)$. Cho [2] has recently given such an injection for $\lambda$ a two part partition, but the given injection does not hold for general $\lambda$ and finding such an injection for all partitions $\lambda$ is left as an open question. In Section 3 of this paper, we give explicit proofs for some known but not well documented results on the charge statistic, $\operatorname{ch}(\pi)$, namely

$$
\sum_{\pi \in W_{\lambda}} q^{i n v(\pi)}=\sum_{\pi \in W_{\lambda}} q^{c h(\pi)}
$$

This implies that

$$
g^{\lambda}(q)-f^{\lambda}(q)=\sum_{\pi \in W_{\lambda}} q^{c h(\pi)}-\sum_{T \in S Y T(\lambda)} q^{\operatorname{maj}(T)} .
$$

The main result of this paper, in Section 4, is to answer Cho's open questions by giving a general injection $h$ from $S Y T(\lambda)$ to $W_{\lambda}$ which takes $\operatorname{maj}(T)$ to $\operatorname{ch}(h(T))$. Section 2 of the paper contains necessary background and definitions.

\section{Definitions and Background}

We say $\lambda=\left(\lambda_{1}, \lambda_{2}, \ldots, \lambda_{k}\right)$ is a partition of $n$ if $\lambda_{1} \geq \lambda_{2} \geq \cdots \geq \lambda_{k}>0$ and $\sum_{i=1}^{k} \lambda_{i}=n$. A partition is described pictorially by its Ferrers diagram, an array of $n$ dots into $k$ leftjustified rows with row $i$ containing $\lambda_{i}$ dots for $1 \leq i \leq k$. For example, the Ferrers diagram for the partition $\lambda=(6,5,3,3,1)$ is:

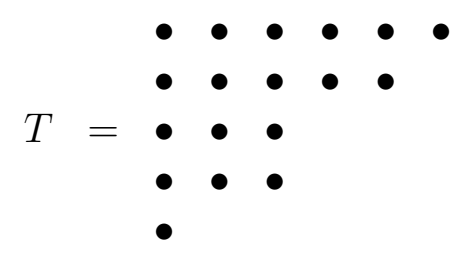

A standard Young tableau of shape $\lambda$ is a filling of the Ferrers diagram for $\lambda$ with the numbers $1,2, \ldots, n$ such that rows are strictly increasing from left to right and columns are strictly increasing from top to bottom. One example of a standard Young tableau for the partition $\lambda=65331$ is shown below:

$T=\begin{array}{cccccc}1 & 2 & 6 & 7 & 9 & 14 \\ 3 & 5 & 8 & 15 & 17 & \\ 4 & 11 & 12 & & & \\ 10 & 16 & 18 & & & \\ 13 & & & & & \end{array}$

Let $f^{\lambda}$ denote the number of standard Young tableaux of shape $\lambda$.

For a standard Young tableau $T$, the major index of $T$ is given by

$$
\operatorname{maj}(T)=\sum_{i \in D(T)} i
$$


where $D(T)=\{i \mid i+1$ is in a row strictly below that of $i$ in $T\}$. For the tableau $T$ given in the previous example, $D(T)=\{2,3,7,9,12,14,15,17\}$ and $\operatorname{maj}(T)=79$.

For a permutation $\pi=\pi_{1} \pi_{2} \cdots \pi_{n} \in S_{n}$, define an inversion to be a pair $(i, j)$ such that $i<j$ and $\pi_{i}>\pi_{j}$. Then the inversion statistic, inv $(\pi)$, is the total number of inversions in $\pi$.

For example, for $\pi=\begin{array}{lllllllll}3 & 2 & 8 & 5 & 7 & 4 & 6 & 1 & 9\end{array}$, inv $(\pi)=15$ since each of the pairs $(1,2),(1,3),(1,4),(1,5),(1,6),(1,7),(1,8),(2,3),(4,5),(4,7),(4,8),(5,8),(6,7),(6,8)$, $(7,8)$ is an inversion.

Let $W_{\lambda}$ be the subset of $S_{n}$ such that

$$
\begin{gathered}
\pi_{1}<\pi_{2}<\cdots<\pi_{\lambda_{1}} \\
\pi_{\lambda_{1}+1}<\pi_{\lambda_{1}+2}<\cdots<\pi_{\lambda_{1}+\lambda_{2}} \\
\cdots \\
\pi_{\lambda_{1}+\lambda_{2}+\cdots+\lambda_{k-1}+1}<\pi_{\lambda_{1}+\lambda_{2}+\cdots+\lambda_{k-1}+2}<\cdots<\pi_{n}
\end{gathered}
$$

For example, for $\lambda=(4,3,3,1)$,

$$
\pi=2 \begin{array}{lllllllllll}
\pi & 5 & 9 & 1 & 3 & 10 & 6 & 8 & 11 & 7
\end{array}
$$

is an element of $W_{4331}$.

We will use the definition of $W_{\lambda}$ for $\lambda$ any combination of $n$, not just for $\lambda$ a partition of $n$. Note that there is no required relationship between $\pi_{\lambda_{1}}$ and $\pi_{\lambda_{1}+1}$, between $\pi_{\lambda_{1}+\lambda_{2}}$ and $\pi_{\lambda_{1}+\lambda_{2}+1}$, and so on. For any $W_{\lambda}=W_{\lambda_{1}, \lambda_{2}, \ldots, \lambda_{k}}$, define $W_{\overline{\lambda_{i}}}=W_{\lambda_{1}, \lambda_{2}, \ldots, \lambda_{i}-1, \ldots, \lambda_{k}}$ for $1 \leq i \leq k$.

Let $\pi$ be a permutation in $S_{n}$. For any $i$ in the permutation, define the charge value of $i, \operatorname{chv}(i)$, recursively as follows:

$$
\begin{aligned}
& \operatorname{chv}(1)=0 \\
& \operatorname{chv}(i)=\operatorname{chv}(i-1) \text { if } i \text { is to the right of } i-1 \text { in } \pi \\
& \operatorname{chv}(i)=\operatorname{chv}(i-1)+1 \text { if } i \text { is to the left of } i-1 \text { in } \pi
\end{aligned}
$$

Now for $\pi \in S_{n}$, define the charge of $\pi, \operatorname{ch}(\pi)$, to be

$$
\operatorname{ch}(\pi)=\sum_{i=1}^{n} \operatorname{chv}(i) .
$$

In the following example of a permutation $\pi=328574619$ with $\operatorname{ch}(\pi)=25$, the charge values of each element are given below the permutation:

$$
\pi=\begin{array}{lllllllll}
3 & 2 & 8 & 5 & 7 & 4 & 6 & 1 & 9 \\
2 & 1 & 5 & 3 & 4 & 2 & 3 & 0 & 5
\end{array}
$$

The definition of the charge statistic was first given by Lascoux and Schützenberger [8]. 
For each element $i \in \pi$, define the charge contribution of $i, c c(i)$, to be zero if $i=1$ or $i$ lies to the right of $i-1$ in $\pi$ and to be $n-i+1$ if $i$ lies to the left of $i-1$ in $\pi$. It is easy to check that $\operatorname{ch}(\pi)=\sum_{i} c c(i)$. For the previous example, the charge contribution of each element is given below that element:

$$
\pi=\begin{array}{lllllllll}
3 & 2 & 8 & 5 & 7 & 4 & 6 & 1 & 9 \\
7 & 8 & 2 & 5 & 3 & 0 & 0 & 0 & 0
\end{array}
$$

\section{Charge and Inv}

Many of the known results on the charge statistic are implicitly given in a number of papers or unpublished manuscripts [1] [5] [6] [7]. The goal of this section is to give explicit proofs of those results which are used in this paper as an aid to the interested reader.

Lascoux and Schützenberger [8] proved the following lemma:

Lemma 1. For $x \in\{2, \ldots, n\}$ and $x \sigma \in S_{n}, \operatorname{ch}(x \sigma)=\operatorname{ch}(\sigma x)+1$.

This result immediately gives

\section{Lemma 2.}

$$
\sum_{\pi \in S_{n}} q^{c h(\pi)}=\left(1+q+q^{2}+\cdots+q^{n-1}\right) \sum_{\sigma \in S_{n-1}} q^{c h(\sigma)} .
$$

Proof. Let $\sigma \in S_{n-1}$, so $\sigma=\sigma_{1} \sigma_{2} \cdots \sigma_{n-1}$. Rewrite $\sigma$ using the numbers $2,3, \ldots, n$ by letting $\tilde{\sigma}_{i}=\sigma_{i}+1$ for every $i$. Let $\pi=1 \tilde{\sigma_{1}} \tilde{\sigma_{2}} \cdots \widetilde{\sigma_{n-1}}$. Then $\pi \in S_{n}$ and $\operatorname{ch}(\pi)=\operatorname{ch}(\sigma)$. By Lemma 1 ,

$$
\begin{aligned}
\operatorname{ch}\left(\widetilde{\sigma_{n-1}} 1 \tilde{\sigma_{1}} \tilde{\sigma_{2}} \cdots \widetilde{\sigma_{n-2}}\right) & =\operatorname{ch}(\pi)+1 \\
& =\operatorname{ch}(\sigma)+1 .
\end{aligned}
$$

Similarly,

$$
\begin{aligned}
\operatorname{ch}\left(\widetilde{\sigma_{n-2}} \widetilde{\sigma_{n-1}} 1 \tilde{\sigma_{1}} \cdots \widetilde{\sigma_{n-3}}\right) & =\operatorname{ch}(\pi)+2 \\
& =\operatorname{ch}(\sigma)+2 \\
& \cdots \\
\operatorname{ch}\left(\tilde{\sigma_{1}} \tilde{\sigma_{2}} \cdots \widetilde{\sigma_{n-1}} 1\right) & =\operatorname{ch}(\pi)+n-1 \\
& =\operatorname{ch}(\sigma)+n-1
\end{aligned}
$$

Thus

$$
\sum_{\pi \in S_{n}} q^{c h(\pi)}=\left(1+q+\cdots+q^{n-1}\right) \sum_{\sigma \in S_{n-1}} q^{c h(\sigma)} .
$$

It is well-known that the inversion statistic satisfies the same recurrence. 


\section{Lemma 3.}

$$
\sum_{\pi \in S_{n}} q^{i n v(\pi)}=\left(1+q+q^{2}+\cdots+q^{n-1}\right) \sum_{\sigma \in S_{n-1}} q^{i n v(\sigma)}
$$

Proof. For details about the inversion statistic, one can consult [3] or [4].

The following theorem [7] follows immediately from the previous Lemmas once the initial conditions are checked.

\section{Theorem 1.}

$$
\sum_{\pi \in S_{n}} q^{c h(\pi)}=\sum_{\pi \in S_{n}} q^{i n v(\pi)}
$$

We now give details that the charge statistic and the inversion statistic not only have the same generating function on $S_{n}$, but they in fact have the same generating function on $W_{\lambda}$.

Lemma 4. For $\lambda=\left(\lambda_{1}, \lambda_{2}, \ldots \lambda_{k}\right)$ a combination of $n$ for any integer $n$,

$$
\sum_{\pi \in W_{\lambda_{1}, \lambda_{2}, \ldots, \lambda_{k}}} q^{i n v(\pi)}=\sum_{\sigma \in W_{\lambda_{2}, \lambda_{3}, \ldots, \lambda_{k}, \lambda_{1}}} q^{i n v(\sigma)} .
$$

Proof. Let $\pi=\pi_{1} \pi_{2} \ldots \pi_{n} \in W_{\lambda_{1}, \lambda_{2}, \ldots, \lambda_{k}}$. Create $\sigma=\sigma_{1} \sigma_{2} \ldots \sigma_{k} \in W_{\lambda_{2}, \lambda_{3}, \ldots, \lambda_{k}, \lambda_{1}}$ in the following manner. For $1 \leq i \leq \lambda_{1}$, let $\sigma_{n+1-i}=n+1-\pi_{i}$. Next, relabel the elements $\pi_{\lambda_{1}+1}$ through $\pi_{n}$ with the remaining $n-\lambda_{1}$ numbers, in the same relative order. For example, if

$$
\pi=\begin{array}{lllllllllllllll}
\pi & 7 & 11 & 3 & 6 & 1 & 10 & 12 & 15 & 5 & 8 & 14 & 4 & 9 & 13
\end{array}
$$

in $W_{3,2,4,3,3}$, we have

$$
\begin{gathered}
\sigma_{15}=16-\pi_{1}=14 \\
\sigma_{14}=16-\pi_{2}=9 \\
\sigma_{13}=16-\pi_{3}=5
\end{gathered}
$$

and the numbers

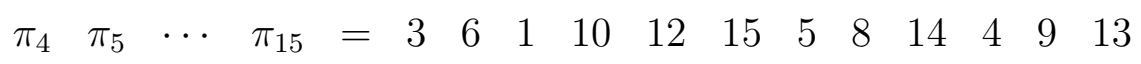

are relabeled in the same relative order using the numbers $[n]-\{5,9,14\}$ to give

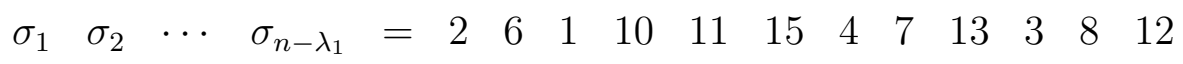

and $\sigma \in W_{2,4,3,3,3}$. Thus

$$
\sigma=2 \begin{array}{lllllllllllllll}
\sigma & 6 & 1 & 10 & 11 & 15 & 4 & 7 & 13 & 3 & 8 & 12 & 5 & 9 & 14 .
\end{array}
$$


It is easy to see that $\sigma$ is unique and that one can reverse the process to take any $\sigma \in W_{\lambda_{2}, \lambda_{3}, \ldots, \lambda_{k}, \lambda_{1}}$ to a unique $\pi \in W_{\lambda_{1}, \lambda_{2}, \ldots, \lambda_{k}}$, so this process gives a bijection between $W_{\lambda_{1}, \lambda_{2}, \ldots, \lambda_{k}}$ and $W_{\lambda_{2}, \lambda_{3}, \ldots, \lambda_{k}, \lambda_{1}}$.

Now we prove that $i n v(\pi)=\operatorname{inv}(\sigma)$. Since $\pi \in W_{\lambda_{1}, \lambda_{2}, \ldots, \lambda_{k}}$, we have $\pi_{1}<\pi_{2}<$ $\cdots<\pi_{\lambda_{1}}$ so there are no inversions between elements $\pi_{1}, \pi_{2}, \cdots, \pi_{\lambda_{1}}$. Similarly, since $\sigma_{n+1-i}=n+1-\pi_{i}$ we have $\sigma_{n-\lambda_{1}+1}<\sigma_{n-\lambda_{1}+2}<\cdots<\sigma_{n}$ so there are no inversions between elements in $\sigma_{n-\lambda_{1}+1}, \sigma_{n-\lambda_{1}+2}, \cdots, \sigma_{n}$. Since $\sigma_{1} \sigma_{2} \cdots \sigma_{n-\lambda_{1}}$ are in the same relative order as $\pi_{\lambda_{1}+1} \pi_{\lambda_{1}+2} \cdots \pi_{n}$, the number of inversions between elements in these two parts is the same.

Now suppose that $\pi_{i}=j$ for $1 \leq i \leq \lambda_{1}$. Then $\pi_{i}$ forms inversions with $(j-1)-(i-1)=$ $j-i$ elements in $\pi_{\lambda_{1}+1} \pi_{\lambda_{1}+2} \cdots \pi_{n}$ since there are $j-1$ total elements less than $j$ and $i-1$ of them lie to the left of $\pi_{i}$ in $\pi$. If $\pi_{i}=j$ then $\sigma_{n+1-i}=n+1-j$. There are $j-1$ total elements bigger than $n+1-j$ and $i-1$ of them lie to the right of $\sigma_{n+1-j}$ in $\sigma$ since there $i-1$ elements to the left of $\pi_{i}=j$ in $\pi$. This means that $\sigma_{n+1-j}$, like $\pi_{i}$, forms inversions with $(j-1)-(i-1)=j-i$ elements in $\sigma_{1} \sigma_{2} \cdots \sigma_{n-\lambda_{1}}$.

Lemma 5. For $\lambda=\left(\lambda_{1}, \lambda_{2}, \ldots \lambda_{k}\right)$ a combination of $n$ for any integer $n$,

$$
\begin{aligned}
\sum_{\pi \in W_{\lambda}} q^{i n v(\pi)}= & \left(\sum_{\sigma \in W_{\bar{\lambda}_{1}}} q^{i n v(\sigma)}\right)+\left(q^{\lambda_{1}} \sum_{\sigma \in W_{\bar{\lambda}_{2}}} q^{i n v(\sigma)}\right)+\cdots \\
& +\left(q^{\lambda_{1}+\lambda_{2}+\cdots+\lambda_{k-1}} \sum_{\sigma \in W_{\lambda_{\bar{k}}}} q^{i n v(\sigma)}\right) .
\end{aligned}
$$

Proof. Again, for the details of results on the inversion statistic, one can consult [3] or [4].

Lemma 6. For $\lambda=\left(\lambda_{1}, \lambda_{2}, \ldots \lambda_{k}\right)$ a combination of $n$ for any integer $n$,

$$
\begin{aligned}
\sum_{\pi \in W_{\lambda}} q^{c h(\pi)}= & \left(\sum_{\sigma \in W_{\lambda_{1}-1, \lambda_{2}, \ldots, \lambda_{k}}} q^{c h(\sigma)}\right)+\left(q^{\lambda_{1}} \sum_{\sigma \in W_{\lambda_{2}-1, \lambda_{3}, \ldots, \lambda_{k}, \lambda_{1}}} q^{c h(\sigma)}\right)+\cdots \\
& +\left(\left(q^{\lambda_{1}+\cdots+\lambda_{k-1}}\right) \sum_{\sigma \in W_{\lambda_{k}-1, \lambda_{1}, \ldots, \lambda_{k-1}}} q^{c h(\sigma)}\right) .
\end{aligned}
$$

Proof. Let $\pi \in W_{\lambda}$. Suppose the 1 in $\pi$ lies in block $\lambda_{i}$, so

$$
\pi=\pi_{1} \pi_{2} \cdots \pi_{\lambda_{1}+\lambda_{2}+\cdots+\lambda_{i-1}} 1 \pi_{\lambda_{1}+\lambda_{2}+\cdots+\lambda_{i-1}+2} \cdots \pi_{n} .
$$

By Lemma 1,

$$
\operatorname{ch}(\pi)=\operatorname{ch}\left(1 \pi_{\lambda_{1}+\lambda_{2}+\cdots+\lambda_{i-1}+2} \cdots \pi_{n} \pi_{1} \pi_{2} \cdots \pi_{\lambda_{1}+\lambda_{2}+\cdots+\lambda_{i-1}}\right)+\lambda_{1}+\lambda_{2}+\cdots+\lambda_{i-1} .
$$


To form $\sigma \in W_{\lambda_{i}-1, \lambda_{i+1}, \ldots, \lambda_{k}, \lambda_{1}, \ldots, \lambda_{i-1}}$, we now remove the initial 1 and relabel each of the remaining $\pi_{i}$ with $\pi_{i}-1$. Since we have removed an initial 1 , the charge of

$$
1 \pi_{\lambda_{1}+\lambda_{2}+\cdots+\lambda_{i-1}+2} \cdots \pi_{n} \pi_{1} \pi_{2} \cdots \pi_{\lambda_{1}+\lambda_{2}+\cdots+\lambda_{i-1}}
$$

is equal to the charge of the newly formed $\sigma$. Thus for each $\pi \in W_{\lambda}$ with a 1 in the $\lambda_{i}$ block and $\sigma$ formed in this manner,

$$
\operatorname{ch}(\pi)=\operatorname{ch}(\sigma)+\left(\lambda_{1}+\lambda_{2}+\cdots+\lambda_{i-1}\right) .
$$

which gives the desired result.

Theorem 2. For $\lambda=\left(\lambda_{1}, \lambda_{2}, \ldots \lambda_{k}\right)$ a combination of $n$ for any integer $n$,

$$
\sum_{\pi \in W_{\lambda}} q^{i n v(\pi)}=\sum_{\pi \in W_{\lambda}} q^{c h(\pi)}
$$

Proof. This result follows immediately by induction from Lemmas 4, 5 and 6 .

\section{An Injection from $S Y T(\lambda)$ to $W_{\lambda}$}

From Section 1, we have that $g^{\lambda}(q)-f^{\lambda}(q)=\sum_{\pi \in W_{\lambda}} q^{\operatorname{ch}(\pi)}-\sum_{\pi \in S Y T(\lambda)} q^{\operatorname{maj}(T)}$ is a polynomial with non-negative coefficients. We will now define an injection $h$ from $S Y T(\lambda)$ to $W_{\lambda}$ such that $\operatorname{maj}(T)=c h(h(T))$. Let $T \in S Y T(\lambda)$. Write down the elements in $T$ by first reading the top row of $T$ from right to left, then the second row of $T$ from right to left, and so on until reaching the bottom row. Call this permutation $\sigma$. For example, if

$$
T=\begin{array}{llll}
1 & 2 & 3 & 6 \\
4 & 8 & 9 & \\
5 & & & \\
7 & & &
\end{array}
$$

then $\sigma=632198457$. To create $\pi \in W_{\lambda}$, let $\pi_{i}=n-\sigma_{i}+1$. In the example, $\pi=478912653$ and $\pi \in W_{4311}$. Let $h(T)=\pi$. Note that for a given $T, h(T)$ is uniquely defined. Since each row of $T$ is strictly increasing, then the first $\lambda_{1}$ elements of $\sigma$ are strictly decreasing, the next $\lambda_{2}$ elements of $\sigma$ are strictly decreasing, and so on. Thus when $\pi$ is formed, the first $\lambda_{i}$ elements of $\pi$ are strictly increasing, the next $\lambda_{2}$ elements of $\pi$ are strictly increasing, and so on, so $\pi \in W_{\lambda}$.

Theorem 3. For $T \in S Y T(\lambda), \operatorname{maj}(T)=\operatorname{ch}(h(T))$.

Proof. We will prove that if $i \in D(T)$, then the charge contribution of $n-i+1$ in $h(T)$ is equal to $i$. In addition, if $i$ is not in $D(T)$, then the charge contribution of $n-i+1$ in $h(T)$ is equal to 0 .

Let $i \in D(T)$. Then $i$ lies in a row strictly above that of $i+1$ in $T$. This implies that $i$ lies to the left of $i+1$ in $\sigma$, and thus $n-i+1$ lies to the left of $n-(i+1)+1=n-i$ 
in $\pi$. By the definition of charge contribution, we find that since $n-i+1$ lies to the left of $n-i$ the charge contribution of $n-i+1$ is equal to $n-(n-i+1)-1=i$.

Suppose $i \notin D(T)$. Then $i$ either lies in a row below $i+1$ in $T$ or they lie in the same row, in which case $i$ lies to the left of $i+1$. In either case, $i$ lies to the right of $i+1$ in $\sigma$ and thus $n-i+1$ lies to the right of $n-(i+1)+1=n-i$ in $\pi$. By the definition of charge contribution, we find that the charge contribution of $n-i+1$ is equal to zero.

Since $\operatorname{maj}(T)=\sum_{\{i \in D(T)\}} i$ and $c h(\pi)=\sum_{i} c c(i)$, we have that $\operatorname{maj}(T)=\operatorname{ch}(h(T))$.

In the previous example, $D(T)=\{3,4,6\}$ so $\operatorname{maj}(T)=13$ and $\operatorname{ch}(h(T))=\operatorname{ch}(478912653)$ which is also 13 .

\section{References}

[1] L. Butler, Subgroup Lattices and Symmetric Functions, Mem. Amer. Math. Soc. 112 No. 539 (1994) vi +160.

[2] S. Cho, Major Index for Standard Young Tableaux, ARS Combinatoria 71 (2004) pp. 93-99.

[3] D. Foata and M. Schützenberger, Major index and inversion number of permutations, Math. Nachr. 83 (1978) 143-159.

[4] I. Goulden and D. Jackson, Combinatorial Enumeration, Dover Publications, 2004.

[5] K. Killpatrick, A Combinatorial Proof of a Recursion for the $q$-Kostka Polynomials, Journal of Combinatorial Theory, Ser. A 92 No. 1 (2000) 29-53.

[6] K. Killpatrick, Recursions for the q-Kostka Polynomials, Ph.D. thesis, University of Minnesota, 1998.

[7] A. Lascoux and Schützenberger, M. P., Sur une conjecture de H. O. Foulkes, C.R. Acad. Sc. Paris 286A (1978) 323-324.

[8] A. Lascoux and Schützenberger, M. P., Croissance des polynomes de Foulkes-Green, C. R. Acad. Sci. Paris 228 (1979) 95-98.

[9] I.G. Macdonald, Symmetric functions and Hall polynomials, Oxford University Press, 1995. 